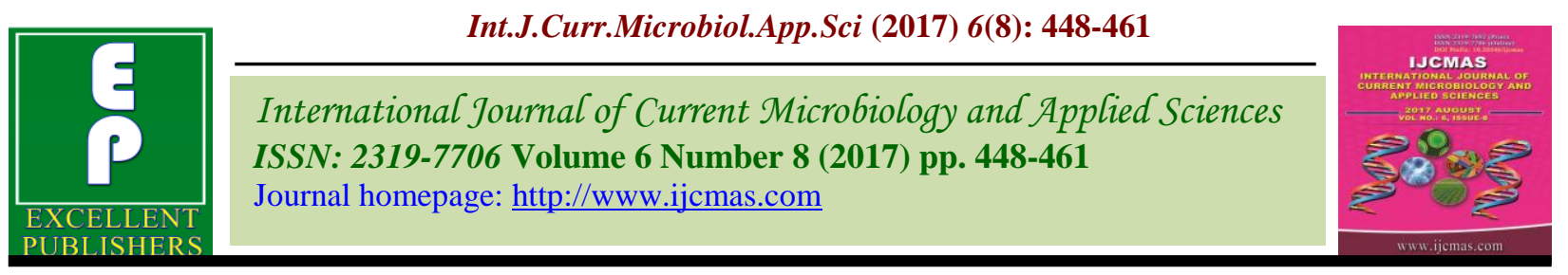

Original Research Article

https://doi.org/10.20546/ijcmas.2017.608.059

\title{
Development of Superior Hybrids for Fibre Quality based on Heterosis and Combining Ability in Upland Cotton (Gossypium hirsutum L.)
}

\author{
Sukhdeep Singh Sivia ${ }^{*}$, S.S. Siwach, O. Sangwan and Sunayana \\ Department of Genetics and Plant Breeding, CCS Haryana Agricultural University, \\ Hisar-125 004, India \\ *Corresponding author
}

\begin{abstract}
A B S T R A C T
The cultivated Gossypium spp. represents the most important, natural fibre crop in the world. Breeding for high cotton yield is still the primary goal of cotton breeding programs, but improving fibre quality has become increasingly important. The enhancement of fibre quality traits like fibre length, strength, and fibre fineness is an essential requirement for the modern textile industry. The objective of this study was to facilitate the selection in cotton breeding program and estimate the general combining ability (GCA) of the parents and specific combining ability (SCA) of hybrids considered for the development of high yielding and better fiber quality in early generations. The study was carried out at cotton research area, CCS Haryana Agricultural University, Hisar (India) during 2014 and 2015 kharif season. Fifteen cotton lines (which have maximum diversity) and four testers (which are known as well adapted and high yielding) were crossed in a line $\mathrm{x}$ tester mating design in 2014. Nineteen genotypes and $60 F_{1}$ hybrids were planted in the randomized complete block design with three replications at the same experimental area in 2015. The ratio of $\delta^{2}$ GCA $/ \delta^{2}$ SCA was less than unity for all the nine characters indicating preponderance of non-additive gene action (dominance and epistasis), which is an important in exploitation of heterosis through hybrid breeding. The best general combining ability was detected from the parent H1470 for seed cotton yield, H1464 and H1098-i for fiber quality traits. SCA was significant for AC726 x H1236, ISR12 x H1226, HR1 x H1117 hybrid combinations for yield and fiber quality. The crosses H1470 x H1236 and H1470 x H1098i were reported good heterosis for seed cotton yield as well as for fibre quality, selected the best hybrids were H1464 x H1098-i and H1463 x H1226. These cross combinations involved at least one parent with high or average GCA effect for a particular trait. The cross combination involving H1470, H1098-i and H1464 parents' recorded significant positive heterosis with acceptable SCA effect for both yield and fibre quality parameters. This investigation concluded that the parents H1470, H1098-i and H1464 can be used in hybrid development programme with better fibre quality.
\end{abstract}

\section{Introduction}

Cotton (Gossypium hirsutum L.) is an important fibre crop and plays a vital role in commerce of many countries such as USA, China, India, Pakistan, Uzbekistan, Turkey, Australia, Greece, Brazil, Egypt etc., where climatic conditions suits its growth, which includes periods of hot and dry weather and adequate moisture. Cotton is harvested as 'seed cotton', which is then 'ginned' to separate the seed and lint. The long 'lint' 
fibres are further processed by spinning to produce yarn that is knitted or woven into fabrics. Although, Indian cottons have very wide quality spectrum, the right combination of fibre length, micronaire and fibre strength is however absent in many of the popular varieties and hybrids. The deficiency in particularly discernable in the staple range of 27 to $30 \mathrm{~mm}$ combined with micronaire of 4.0 to 4.5 and a strength of 22 to $25 \mathrm{~g}$ per tex. There is an urgent need to promote those cottons that could come closer in quality to compete in the international market. For this purpose, development of superior hybrids for seed cotton yield with desirable fibre quality.

Exploitation of hybrid vigour has become potential tool for the improvement of fibre quality in this crop. However, lot of information is available on heterosis in cotton but still it holds future promise for further utilization. Hybridization is the most potent technique for breaking undesirable linkages between yield and fibre traits. The choice of suitable parents for the development of desired hybrid depends on the selection of parents based on combining ability. To study the extent of heterosis and combining ability of a number of parents, Line $x$ Tester analysis is the most appropriate procedure. Based on the information from Line $\mathrm{x}$ Tester analysis production of commercially viable hybrid is possible. The purposes of this study were to find out the extent of useful heterosis as well as GCA and SCA effects over the check hybrid $\mathrm{HHH} 223$ for fibre quality and seed cotton yield.

\section{Materials and Methods}

\section{Selection of parental line}

The parental lines to be used in the present study were selected based on their genetic divergence. Large number of genetic accessions studied and selected fifteen diverse female lines viz., H1156, ISR12, HR1, Luxmi
PKV, AC726, Deltapine, H1472, H1465, H1463, H1464, H1470, H1471, H1476, H1477 and CSH3075 during the Kharif season of 2013. The male lines was selected based on their agronomical superiority and selected four local cultivars, viz., H1226, H1098-I, H1117 and H1236.

\section{Hybrid development}

All the diverse parental lines (15) were crossed with all the four male parents in Line $\mathrm{x}$ Tester fashion during the Kharif season of 2014. When the parental lines started to flower, these were crossed in line $\mathrm{x}$ tester fashion. Some of the buds of parents were also selfed. Maximum numbers of crosses were made to develop sufficient $F_{1}$ seed. The following necessary precautions were taken at the time of emasculation and pollination: (1) Emasculation was done before the anthers are mature and the stigma has become receptive to minimize self-pollination. (2) The flowers selected for emasculation are likely to open the next morning. (3) Care was taken that all the anthers are removed. (4) The gynoecium must not be injured and (5) Bagging of emasculated buds before and after pollination.

\section{Field layout}

The 60 hybrids, 19 parents with single check HHH223 were planted in the field during Kharif 2015 crop season at cotton research area, CCS Haryana Agricultural University, Hisar (India). Each entry was sown in randomized block design (RBD) with three replications. Each genotype was grown in a $7.2 \mathrm{~m}$ length row adopting a spacing of 67.5 $\mathrm{cm}$ between rows and $60 \mathrm{~cm}$ between the plants in a row, to have 13 plants per row.

\section{Data analysis}

Data were recorded on five randomly selected plants per replication for all the nine quantitative and qualitative characters viz., 
seed cotton yield per plant (g), lint yield per plant $(\mathrm{g})$, ginning outturn $(\%)$, seed index $(\mathrm{g})$, lint index $(\mathrm{g}), 2.5 \%$ span length $(\mathrm{mm})$, fibre uniformity $(\%)$, fibre strength $(\mathrm{g} / \mathrm{tex})$ and micronaire value ( $\mu \mathrm{g} / \mathrm{inch})$.

\section{Statistical analysis}

The mean values of the characters measured in 80 genotypes in each replication were analyzed for analysis of variance, estimation of standard error and critical difference by adopting the method suggested by Panse and Sukhatme (1961). The Line x Tester analysis of combining ability analysis of the data was done as suggested by Kempthorne (1957).

\section{Results and Discussion}

The analysis of variance (Table 1) indicated that the mean squares of genotypes for all the characters investigated were significantly different, indicating the presence of variability among hybrids and their parents. The information on combining ability will help the breeder in developing the future breeding programme to be adopted for exploiting additive and/or non-additive components present in the material. In the present investigation, an attempt was made to obtain information on the magnitude of GCA and SCA variances and GCA and SCA effects for individual parents and crosses in respect of nine characters through combining ability analysis. Estimates of variances due to general and specific combining ability for all characters under study are presented in table 2. The SCA variances $\left(\delta^{2}\right.$ SCA) were higher than gca variance $\left(\delta^{2} \mathrm{GCA}\right)$ for almost all the characters. The ratio of $\delta^{2}$ GCA $/ \delta^{2}$ SCA was less than unity for all the nine characters indicating preponderance of non-additive gene action (dominance and epistasis), which is an important in exploitation of heterosis through hybrid breeding. Several authors Ahuja and Dhayal (2007), Nidagundi et al.,
(2011) and Pushpam et al., (2015) have reported the predominance of SCA variance in upland cotton for yield and fibre quality characters.

The estimates of general combining ability (GCA) effects of all the parents comprising fifteen female parents and four male parents for all nine characters investigated are presented in table 3. Best combining male and female parents along with the poorest combiners for various characters are presented in table 4 . The perusal of table revealed that among the four male parents, H1236 was the best combiner for the characters viz. seed cotton yield, lint yield, ginning outturn, seed index and lint index. Male parent H1098-i was best combiner for $2.5 \%$ span length, fibre uniformity and fibre strength. H1226 was the best combiner for micronaire value. Male parent H1117 was second best combiner for fibre uniformity.

Among female parents, H1470 was good general combiner for seed cotton yield, lint yield and the same parent also second best combiner for $2.5 \%$ span length and fibre uniformity. The genotype H1471 was found good combiner ginning out turn and lint index. Female parent H1464 was found best combiner for seed index, $2.5 \%$ span length, fibre uniformity and fibre strength. H1463 was also found to be good combiner for micronaire value. Similar results were reported by Kumar et al., (2013), Deshmukh et al., (2014) and Kencharaddi et al., (2015). Female parent H1156 was observed the second best combiner for fibre strength and micronaire value. The poor GCA effect was obtained from parent AC726 for $2.5 \%$ span length, fibre uniformity and fibre strength. Female parent HR1 was recorded poor general combiner for ginning outturn, seed index and lint index. Parent CSH3075 had second best GCA effect for ginning outturn whereas poor combiner for micronaire value. 
The second best GCA effect was reported from $\mathrm{H} 1465$ for seed index and lint index. However, considering the economic importance of various characters, parents H1470, H1464, H1098-i can be used in future breeding program for improvement of fiber quality parameters.

The estimates of specific combining ability effects are provided in table 5. Best cross combinations for different characters have been presented in table 6 . The table revealed that cross AC726 x H1236 followed by H1476 x H1226 was the top specific combiners for the seed cotton yield per plant. These crosses were combination of both good combining parent, indicating that additive variance were important for this character. Both these crosses were also found to be the top specific cross combination for lint yield per plant. Similar results were reported by Khan et al., (2009) and Alkuddsi et al., (2013).

The cross ISR12 x H1236 exhibited top SCA for seed index and reported significant SCA effect for lint index whereas the cross was combinations of good $\mathrm{x}$ poor combining parents, hence SCA effect of these cross is due to additive and non-additive action. Similar results were reported by Subramanian et al., (2005) and Alkuddsi et al., (2013). The cross combination H1463 x H1236 observed highest SCA effects for ginning out turn and this crosses was combinations of poor $\mathrm{x}$ good combining parents; hence, SCA effect of these crosses is due to both additive and nonadditive gene action. Similar results were reported by Karademir et al., (2007), Anandan (2010) and Rajamani et al., (2014).

Table.1 Mean squares for characters under study

\begin{tabular}{|c|c|c|c|c|c|c|c|c|c|c|}
\hline $\begin{array}{c}\text { Source of } \\
\text { variation }\end{array}$ & df & $\begin{array}{c}\text { Seed } \\
\text { cotton } \\
\text { yield/plant } \\
(\mathbf{g})\end{array}$ & $\begin{array}{c}\text { Lint } \\
\text { yield/plant } \\
(\mathbf{g})\end{array}$ & $\begin{array}{c}\text { Ginning } \\
\text { outturn } \\
(\mathbf{\%})\end{array}$ & $\begin{array}{c}\text { Seed } \\
\text { index } \\
(\mathbf{g})\end{array}$ & $\begin{array}{c}\text { Lint } \\
\text { index } \\
(\mathbf{g})\end{array}$ & $\begin{array}{c}\mathbf{2 . 5 \%} \\
\mathbf{s p a n} \\
\mathbf{l e n g t h} \\
(\mathbf{m m})\end{array}$ & $\begin{array}{c}\text { Fibre } \\
\text { uniformity } \\
(\boldsymbol{\%})\end{array}$ & $\begin{array}{c}\text { Fibre } \\
\text { strength } \\
(\mathbf{g} / \mathbf{t e x})\end{array}$ & $\begin{array}{c}\text { Micronaire } \\
\text { value } \\
(\boldsymbol{\mu g} / \mathbf{i n c h})\end{array}$ \\
\hline Replication & 2 & 123.02 & 24.33 & 7.81 & 0.03 & 0.17 & 0.21 & 1.13 & 0.23 & 0.01 \\
\hline Treatment & 79 & $787.15^{*}$ & $84.31^{*}$ & $3.02^{*}$ & $0.71^{*}$ & $0.33^{*}$ & $4.95^{*}$ & $1.21^{*}$ & $6.12^{*}$ & $0.58^{*}$ \\
\hline Error & 158 & 50.24 & 5.94 & 0.58 & 0.03 & 0.02 & 0.17 & 0.39 & 0.02 & 0.03 \\
\hline C.D. & & 11.44 & 3.93 & 1.23 & 0.26 & 0.23 & 0.66 & 1.01 & 0.46 & 0.26 \\
\hline C.V. & & 10.33 & 10.88 & 2.20 & 2.50 & 4.15 & 1.64 & 0.78 & 1.11 & 4.08 \\
\hline
\end{tabular}

*Significant at $5 \%$ level of significance

Table.2 Combining ability analysis for sixteen characters in Gossypium hirsutum L.

\begin{tabular}{|c|c|c|c|c|c|c|c|c|c|c|}
\hline $\begin{array}{l}\text { Source of } \\
\text { variation }\end{array}$ & df & $\begin{array}{c}\text { Seed cotton } \\
\text { yield/plant } \\
\text { (g) }\end{array}$ & $\begin{array}{c}\text { Lint } \\
\text { yield/plant } \\
\text { (g) }\end{array}$ & $\begin{array}{c}\text { Ginning } \\
\text { outturn } \\
(\%)\end{array}$ & $\begin{array}{c}\text { Seed } \\
\text { index } \\
(\mathrm{g})\end{array}$ & $\begin{array}{c}\text { Lint } \\
\text { index } \\
(\mathrm{g})\end{array}$ & $\begin{array}{c}2.5 \% \\
\text { span } \\
\text { length } \\
(\mathrm{mm})\end{array}$ & $\begin{array}{c}\text { Fibre } \\
\text { uniformity } \\
(\%)\end{array}$ & $\begin{array}{c}\text { Fibre } \\
\text { strength } \\
(\mathrm{g} / \mathrm{tex})\end{array}$ & $\begin{array}{c}\text { Micronaire } \\
\text { value } \\
(\mu \mathrm{g} / \text { inch })\end{array}$ \\
\hline Replication & 2 & 83.98 & $25.57 *$ & $4.14 * *$ & 0.04 & $0.09 *$ & .23 & .45 & .19 & .01 \\
\hline Hybrid & 59 & $872.82^{* *}$ & $92.02 * *$ & $3.17 * *$ & $.76^{* * *}$ & $.36^{* * *}$ & 4.90 & 1.10 & 6.38 & 0.58 \\
\hline Lines & 14 & $2043.29 * *$ & $212.65 * *$ & $4.19 * *$ & 1.69 ** & $0.74 * *$ & 9.79 & 2.37 & 9.87 & 0.95 \\
\hline Testers & 3 & $508.978 * *$ & $61.806 * *$ & $1.316^{*}$ & $0.45^{* *}$ & $0.292 * *$ & 4.24 & 1.71 & 3.02 & 0.03 \\
\hline $\begin{array}{l}\text { Lines } x \\
\text { Testers }\end{array}$ & 42 & $508.66^{* *}$ & $53.97 * *$ & $2.97 * *$ & $0.47 * *$ & $0.24 * *$ & 3.31 & 0.64 & 5.45 & 0.50 \\
\hline Error & 118 & 49.65 & 5.93 & 0.60 & 0.02 & 0.02 & 0.18 & 0.39 & 0.09 & 0.02 \\
\hline$\sigma^{2}$ gca & & 26.93 & 2.92 & 0.01 & 0.02 & 0.01 & 0.13 & 0.05 & 0.04 & 0.02 \\
\hline$\sigma^{2}$ sca & & 1888.68 & 199.61 & 6.96 & 1.75 & 0.83 & 11.88 & 1.68 & 16.77 & 1.42 \\
\hline$\sigma^{2} \mathbf{g c a} / \sigma^{2} \mathbf{s c a}$ & & 0.03 & 0.03 & 0.00 & 0.02 & 0.02 & 0.011 & 0.03 & 0.00 & 0.01 \\
\hline
\end{tabular}

$* *$ Significant at $1 \%$ level of significance; *Significant at $5 \%$ level of significance. 
Table.3 General combining ability effects of parents for different characters in Gossypium hirsutum L.

\begin{tabular}{|c|c|c|c|c|c|c|c|c|c|}
\hline Female & $\begin{array}{c}\text { Seed } \\
\text { cotton } \\
\text { yield/plant } \\
(\mathrm{g})\end{array}$ & $\begin{array}{c}\text { Lint } \\
\text { yield/plant } \\
(\mathrm{g})\end{array}$ & $\begin{array}{c}\text { Ginning } \\
\text { outturn } \\
(\%)\end{array}$ & $\begin{array}{c}\text { Seed } \\
\text { index }(g)\end{array}$ & $\underset{\text { index }(g)}{\operatorname{Lint}}$ & $\begin{array}{c}2.5 \% \\
\text { span } \\
\text { length } \\
(\mathrm{mm})\end{array}$ & $\begin{array}{c}\text { Fibre } \\
\text { uniformity } \\
(\%)\end{array}$ & $\begin{array}{c}\text { Fibre } \\
\text { strength } \\
\text { (g/tex) }\end{array}$ & $\begin{array}{c}\text { Micronaire } \\
\text { value } \\
\text { ( } \mu \text { g/inch) }\end{array}$ \\
\hline H1156 & -3.58 & -1.24 & -0.11 & 0.05 & 0.01 & $-0.64 * *$ & -0.25 & $0.57 * *$ & $0.31 * *$ \\
\hline ISR12 & $-9.31 * *$ & $-3.31 * *$ & -0.41 & $-0.18^{* *}$ & $-0.16^{* *}$ & 0.02 & 0.02 & 0.19 & $-0.19 * *$ \\
\hline HR1 & 0.22 & -0.62 & $-1.05 * *$ & $-0.64 * *$ & $-0.49 * *$ & $-1.19 * *$ & $-0.83 * *$ & $-0.66 * *$ & $0.26 * *$ \\
\hline $\begin{array}{l}\text { Luxmi } \\
\text { PKV }\end{array}$ & $-13.83 * *$ & $-4.47 * *$ & 0.01 & $-0.35^{* *}$ & $-0.19 * *$ & $0.59 * *$ & 0.25 & $-0.45 * *$ & $-0.15^{*}$ \\
\hline AC726 & 1.71 & 0.45 & -0.37 & $-0.43 * *$ & $-0.28 * *$ & $-2.26^{* *}$ & $-1.00 * *$ & $-2.15^{* *}$ & $-0.34 * *$ \\
\hline Delta Pine & $-20.35 * *$ & $-6.76 * *$ & -0.17 & $-0.54 * *$ & $-0.31 * *$ & $-0.45^{* *}$ & -0.08 & $-0.33 * *$ & 0.01 \\
\hline H1472 & 0.43 & 0.27 & 0.24 & $0.19 * *$ & $0.14 *$ & 0.24 & 0.33 & $0.56^{* *}$ & 0.11 \\
\hline H1465 & $5.74 *$ & 1.94 & 0.15 & $0.43 * *$ & $0.25 * *$ & $0.72 * *$ & 0.16 & $-0.29 * *$ & 0.04 \\
\hline H1463 & 3.31 & 0.68 & -0.45 & 0.01 & -0.05 & -0.15 & 0.16 & $0.53 * *$ & $0.62 * *$ \\
\hline H1464 & $11.76^{* * *}$ & $3.60 * *$ & -0.40 & $0.55 * *$ & $0.22 * *$ & $1.42 * *$ & $0.66 * *$ & $1.85 * *$ & $0.21 * *$ \\
\hline H1470 & $34.06^{* * *}$ & $10.83^{* * *}$ & -0.24 & $0.33 * *$ & $0.13^{*}$ & $0.88^{* *}$ & 0.50 & $0.36^{* *}$ & $-0.21 * *$ \\
\hline H1471 & 2.29 & 1.56 & $1.19 * *$ & $0.41 * *$ & $0.41 * *$ & $0.49 * *$ & -0.08 & $0.49 * *$ & 0.05 \\
\hline H1476 & 7.14* & $2.46^{*}$ & 0.14 & $0.18 * *$ & $0.11 *$ & $0.41 *$ & 0.25 & $0.33 * *$ & $-0.32 * *$ \\
\hline H1477 & $-16.27^{* * *}$ & $-5.08 * *$ & 0.35 & $0.13^{*}$ & $0.12 *$ & 0.12 & 0.01 & 0.04 & -0.01 \\
\hline CSH3075 & -3.35 & -0.32 & $1.14 * *$ & $-0.14 *$ & 0.08 & -0.21 & -0.08 & $-1.06^{* *}$ & $-0.38 * *$ \\
\hline SE (d) & 2.87 & 0.99 & 0.31 & 0.06 & 0.05 & 0.17 & 0.25 & 0.11 & 0.06 \\
\hline \multicolumn{10}{|c|}{ Male parents } \\
\hline H1226 & 0.74 & 0.16 & -0.09 & -0.03 & -0.03 & $-0.41 * *$ & -0.25 & $-0.36 * *$ & 0.02 \\
\hline H1098-i & -1.89 & -0.67 & -0.09 & $-0.09 * *$ & $-0.06^{*}$ & $0.25 * *$ & 0.21 & $0.19 * *$ & 0.01 \\
\hline H1117 & $-3.24 *$ & $-1.07 *$ & -0.06 & -0.01 & -0.02 & $0.21^{*}$ & 0.06 & $0.16^{* * *}$ & 0.01 \\
\hline H1236 & $4.39 * *$ & $1.58^{* *}$ & 0.25 & $0.14 * *$ & $0.11 * *$ & -0.05 & -0.02 & 0.01 & -0.03 \\
\hline SE (d) & 1.48 & 0.51 & 0.16 & 0.03 & 0.03 & 0.08 & 0.13 & 0.06 & 0.03 \\
\hline
\end{tabular}

**Significant at $1 \%$ level of significance

*Significant at $5 \%$ level of significance

Table.4 Above average and poorest general combining parents for different characters

\begin{tabular}{|c|c|c|c|c|}
\hline \multirow{3}{*}{ Characters } & \multicolumn{3}{|c|}{ Female parent } & \multirow{3}{*}{$\begin{array}{c}\text { Male parent } \\
\text { Above average } \\
\text { Combiner }\end{array}$} \\
\hline & \multicolumn{2}{|c|}{ Above average combiners } & \multirow{2}{*}{ Poor combiners } & \\
\hline & $1^{\text {st }}$ & $2^{\text {nd }}$ & & \\
\hline Seed cotton yield per plant $(\mathrm{g})$ & H1470 (34.07**) & H1464 (11.77**) & Deltapine $(-20.35 * *)$ & $\mathrm{H} 1236(4.39 * *)$ \\
\hline Lint yield/plant (g) & H1470 $\left(10.83^{* *}\right)$ & H1464 (3.60**) & Deltapine $(-6.77 * *)$ & H1236 (1.58**) \\
\hline Ginning outturn (\%) & H1471 (1.19**) & CSH3075 $\left(1.15^{* *}\right)$ & HR1 $\left(-1.05^{* *}\right)$ & H1236 (0.25) \\
\hline Seed index $(g)$ & H1464 (0.55**) & H1465 (0.43**) & HR1 $\left(-0.65^{* *}\right)$ & H1236 $(0.14 * *)$ \\
\hline Lint index (g) & H1471 $(0.42 * *)$ & H1465 $(0.25 * *)$ & HR1 $(-0.49 * *)$ & H1236 $(0.12 * *)$ \\
\hline $2.5 \%$ span length $(\mathrm{mm})$ & H1464 (1.42**) & H1470 $(0.88 * *)$ & $\mathrm{AC7} 26(-2.26 * *)$ & H1098-I $\left(0.25^{* *}\right)$ \\
\hline Fibre uniformity (\%) & H1464 $\left(0.66^{* *}\right)$ & H1470 (0.50) & $\mathrm{AC726}(-1.00 * *)$ & H1098-I (0.21) \\
\hline Fibre strength $(g /$ tex $)$ & H1464 (1.85**) & H1156 (0.57**) & $\mathrm{AC726}\left(-2.15^{* *}\right)$ & H1098-I $\left(0.19^{* *}\right)$ \\
\hline Micronaire value ( $\mu \mathrm{g} /$ inch) & H1463 $(0.62 * *)$ & H1156 (0.31**) & CSH3075 $(-0.38 * *)$ & H1226 (0.02) \\
\hline
\end{tabular}


Table.5 Specific combining ability effects of hybrids for different characters in Gossypium hirsutum L.

\begin{tabular}{|c|c|c|c|c|c|c|c|c|c|}
\hline Cross & $\begin{array}{c}\text { Seed cotton } \\
\text { yield/plant } \\
(\mathrm{g})\end{array}$ & $\begin{array}{c}\text { Lint } \\
\text { yield/plant } \\
(\mathrm{g})\end{array}$ & $\begin{array}{c}\text { Ginning } \\
\text { outturn }(\%)\end{array}$ & $\begin{array}{l}\text { Seed index } \\
(\mathrm{g})\end{array}$ & $\begin{array}{c}\text { Lint index } \\
\text { (g) }\end{array}$ & $\begin{array}{r}2.5 \% \text { span } \\
\text { length }(\mathrm{mm})\end{array}$ & $\begin{array}{c}\text { Fibre } \\
\text { uniformity } \\
(\%)\end{array}$ & $\begin{array}{c}\text { Fibre } \\
\text { strength } \\
(\mathrm{g} / \mathrm{tex}) \\
\end{array}$ & $\begin{array}{c}\text { Micronaire } \\
\text { value }(\mu \mathrm{g} / \text { inch })\end{array}$ \\
\hline H1156 X H1226 & 4.36 & 2.27 & $1.26^{*}$ & $-0.30 *$ & 0.02 & 0.46 & 0.25 & $1.81 * *$ & $0.34 * *$ \\
\hline H1156 X H 1098-i & 4.95 & 1.52 & 0.02 & $0.32 * *$ & 0.17 & $-0.76 * *$ & -0.55 & $-0.79 * *$ & 0.12 \\
\hline H1156 X H1117 & -6.80 & -2.39 & -0.29 & 0.19 & 0.06 & 0.44 & 0.27 & $-0.55^{*}$ & $-0.60 * *$ \\
\hline H1156 X H1236 & -2.51 & -1.45 & -0.99 & -0.20 & $-0.26^{*}$ & -0.14 & 0.02 & -0.46 & 0.13 \\
\hline ISR12 X H1226 & -9.92 & -2.99 & 0.30 & $0.94 * *$ & $0.54 * *$ & $2.26 * *$ & 0.66 & $2.81 * *$ & $0.62 * *$ \\
\hline ISR12 X H 1098-i & 7.93 & 3.42 & $1.33^{*}$ & 0.06 & 0.22 & $-1.19 * *$ & -0.13 & $-0.54 *$ & 0.02 \\
\hline ISR12 X H1117 & -8.65 & -2.91 & -0.31 & $-0.72 * *$ & $-0.42 * *$ & $-1.15 * *$ & -0.31 & $-1.44 * *$ & -0.02 \\
\hline ISR12 X H1236 & 10.65 & 2.48 & $-1.32 *$ & $-0.27 *$ & $-0.34 * *$ & 0.08 & -0.22 & $-0.82 * *$ & $-0.62 * *$ \\
\hline HR1 X H1226 & 1.08 & 0.01 & -0.51 & 0.18 & 0.03 & $-0.71 *$ & -0.50 & $-0.78 * *$ & 0.05 \\
\hline HR1 X H 1098-i & -2.82 & -0.50 & 0.59 & $-0.35 * *$ & -0.09 & 0.18 & 0.70 & $0.98 * *$ & 0.06 \\
\hline HR1 X H1117 & 6.20 & 1.95 & -0.03 & $0.51 * *$ & $0.26^{*}$ & $2.56^{* *}$ & 0.85 & $2.08 * *$ & -0.12 \\
\hline HR1 X H1236 & -4.46 & -1.45 & -0.05 & $-0.35 * *$ & -0.19 & $-2.03 * *$ & $-1.05 *$ & $-2.29 * *$ & 0.01 \\
\hline Luxmi PKV X H1226 & $24.02 * *$ & $7.79 * *$ & -0.07 & $-0.46 * *$ & $-0.26^{*}$ & 0.39 & 0.41 & -0.29 & -0.02 \\
\hline Luxmi PKV X H 1098-i & $-17.65 * *$ & $-6.10 * *$ & -0.89 & $0.28 *$ & 0.01 & $0.76^{*}$ & -0.05 & $0.56^{*}$ & $0.28 *$ \\
\hline Luxmi PKV X H1117 & -8.41 & -2.51 & 0.65 & $0.38 * *$ & $0.30 * *$ & -0.52 & -0.22 & -0.19 & -0.01 \\
\hline Luxmi PKV X H1236 & 2.05 & 0.82 & 0.31 & -0.20 & -0.06 & -0.62 & -0.13 & -0.07 & $-0.26 *$ \\
\hline AC726 X H1226 & -10.31 & -3.31 & 0.26 & $-0.37 * *$ & -0.17 & -0.34 & 0.33 & 0.01 & -0.22 \\
\hline AC726 X H 1098-i & $-12.54 *$ & $-5.21 * *$ & $-1.87 * *$ & $-0.27 *$ & $-0.39 * *$ & -0.64 & -0.46 & -0.33 & 0.21 \\
\hline AC726 X H1117 & -4.91 & -1.45 & 0.50 & $0.46^{* *}$ & $0.31 * *$ & $0.92 * *$ & 0.35 & 0.47 & 0.08 \\
\hline AC726 X H1236 & $27.76 * *$ & $9.97 * *$ & 1.10 & 0.18 & $0.25^{*}$ & 0.06 & -0.22 & -0.14 & -0.07 \\
\hline Delta Pine X H1226 & 1.07 & 0.46 & 0.09 & -0.05 & -0.01 & -0.25 & 0.08 & $-0.61 *$ & $-0.34 * *$ \\
\hline Delta Pine X H 1098-i & 2.63 & 0.92 & -0.04 & -0.23 & -0.13 & $-1.29 * *$ & -0.05 & -0.18 & $0.42 * *$ \\
\hline Delta Pine X H1117 & 10.19 & 2.77 & -1.04 & -0.04 & -0.16 & 0.28 & -0.56 & $-0.87 * *$ & $-0.32 * *$ \\
\hline Delta Pine X H1236 & $-13.90 *$ & $-4.17 *$ & 0.99 & 0.32 & $0.31 * *$ & $1.25 * *$ & 0.52 & $1.67 * *$ & 0.24 \\
\hline H1472 X H1226 & -8.95 & -2.53 & 0.59 & 0.16 & 0.18 & -0.32 & -0.66 & $1.18 * *$ & 0.20 \\
\hline H1472 X H 1098-i & 3.46 & 1.81 & 0.96 & -0.18 & 0.04 & 0.07 & 0.53 & $-2.14 * *$ & $-0.38 * *$ \\
\hline H1472 X H1117 & 9.62 & 1.67 & $-2.03 * *$ & -0.14 & $-0.39 * *$ & 0.05 & 0.02 & $0.48^{*}$ & 0.08 \\
\hline H1472 X H1236 & -4.13 & -0.95 & 0.48 & 0.16 & 0.17 & 0.19 & 0.11 & $0.47 *$ & 0.09 \\
\hline H1465 X H1226 & -3.73 & -0.51 & 0.96 & 0.17 & $0.26^{*}$ & -0.07 & -0.50 & $1.74 * *$ & $0.54 * *$ \\
\hline
\end{tabular}




\begin{tabular}{|c|c|c|c|c|c|c|c|c|c|}
\hline H1465 X H 1098-i & $12.47 *$ & 3.51 & -0.47 & $0.30^{*}$ & 0.07 & 0.42 & 0.03 & -0.22 & -0.17 \\
\hline H1465 X H1117 & -6.90 & -1.71 & 0.85 & -0.12 & 0.06 & -0.36 & 0.18 & $-0.85^{* *}$ & -0.01 \\
\hline H1465 X H1236 & -1.83 & -1.27 & $-1.33^{*}$ & $-0.35^{* *}$ & $-0.40^{* *}$ & 0.01 & 0.27 & -0.66 ** & $-0.36 * *$ \\
\hline H1463 X H1226 & $11.90 *$ & 2.70 & $-1.53 *$ & 0.15 & -0.16 & -0.05 & 0.16 & $-1.58 * *$ & $0.49 * *$ \\
\hline H1463 X H 1098-i & 10.46 & 2.99 & -0.54 & $-0.73^{* *}$ & $-0.46^{* *}$ & $1.31 * *$ & 0.03 & $1.75^{* *}$ & $-0.29 *$ \\
\hline H1463 X H1117 & $-15.88 * *$ & $-4.87 *$ & 0.29 & 0.13 & 0.10 & -0.01 & -0.14 & $0.58^{*}$ & 0.14 \\
\hline H1463 X H1236 & -6.48 & -0.82 & $1.78^{* *}$ & $0.44 * *$ & $0.52 * *$ & $-1.24 * *$ & -0.05 & $-0.75 * *$ & $-0.34 * *$ \\
\hline H1464 X H1226 & 2.95 & 1.05 & 0.24 & $-0.43^{* *}$ & -0.19 & -0.53 & 0.01 & $-1.60 * *$ & $-0.35 * *$ \\
\hline H1464 X H 1098-i & $-18.64 * *$ & -6.40 ** & -0.56 & $0.34 * *$ & 0.07 & $1.03 * *$ & 0.53 & $1.59 * *$ & 0.12 \\
\hline H1464 X H1117 & 6.39 & 1.24 & -0.89 & -0.11 & -0.20 & 0.07 & -0.31 & $0.52 *$ & 0.23 \\
\hline H1464 X H1236 & 9.29 & $4.10^{*}$ & 1.20 & 0.21 & $0.31 * *$ & -0.58 & -0.22 & $-0.51 *$ & 0.01 \\
\hline H1470 X H1226 & $-19.99 * *$ & $-6.35^{* *}$ & 0.05 & $-0.39 * *$ & -0.19 & $-0.93^{* *}$ & -0.16 & $-1.54 * *$ & $-0.52 * *$ \\
\hline H1470 X H 1098-i & $14.64 *$ & $5.06^{*}$ & 0.29 & $-0.30^{*}$ & -0.11 & 0.03 & -0.30 & $-0.58 *$ & $-0.32 *$ \\
\hline H1470 X H1117 & -9.10 & -1.97 & 1.07 & $0.44 * *$ & $0.41 * *$ & $0.74 *$ & 0.18 & -0.01 & -0.01 \\
\hline H1470 X H1236 & $14.46^{*}$ & 3.27 & $-1.41 *$ & $0.25^{*}$ & -0.10 & 0.15 & 0.27 & $2.14 * *$ & $0.86^{* *}$ \\
\hline H1471 X H1226 & -8.91 & -3.47 & -0.76 & 0.06 & -0.09 & 0.09 & -0.25 & -0.34 & $-0.69 * *$ \\
\hline H1471 X H 1098-i & 7.84 & 2.80 & 0.27 & 0.15 & 0.12 & $0.76^{*}$ & 0.28 & $-0.54 *$ & $-0.42 * *$ \\
\hline H1471X H1117 & $11.47 *$ & 3.88 & 0.12 & $-0.41^{* *}$ & -0.21 & $-1.56^{* *}$ & -0.56 & $-0.57 *$ & $0.52 * *$ \\
\hline H1471 X H1236 & -10.40 & -3.21 & 0.36 & 0.19 & 0.18 & $0.70^{*}$ & 0.52 & $1.47 * *$ & $0.59 * *$ \\
\hline H1476 X H1226 & $26.19 * *$ & $8.17 * *$ & -0.43 & -0.09 & -0.11 & $1.01 * *$ & 0.41 & 0.41 & -0.18 \\
\hline H1476 X H 1098-i & -0.35 & 0.35 & 0.53 & $0.56 * *$ & $0.38 * *$ & 0.44 & -0.05 & $1.91 * *$ & $0.72 * *$ \\
\hline H1476 X H1117 & -7.25 & -2.16 & 0.39 & $-0.41 * *$ & -0.16 & $-1.44 * *$ & -0.22 & $-1.28 * *$ & $-0.43 * *$ \\
\hline H1476 X H1236 & $-18.58 * *$ & $-6.35^{* *}$ & -0.50 & -0.05 & -0.10 & -0.01 & -0.13 & $-1.03 * *$ & -0.09 \\
\hline H1477 X H1226 & -4.42 & -1.37 & -0.30 & 0.21 & 0.07 & -0.26 & 0.01 & $-0.76^{* * *}$ & -0.13 \\
\hline H1477X H 1098-i & -7.96 & -2.35 & 0.77 & -0.18 & 0.02 & $-0.93 * *$ & -0.13 & $-1.29 * *$ & $-0.29 *$ \\
\hline H1477 X H1117 & 9.88 & 2.89 & -0.50 & 0.19 & 0.02 & $-0.72^{*}$ & -0.31 & $0.90^{* *}$ & $0.51 * *$ \\
\hline H1477 X H1236 & 2.50 & 0.83 & 0.03 & -0.22 & -0.12 & $1.91 * *$ & 0.44 & $1.15^{* *}$ & -0.08 \\
\hline CSH3075 X H1226 & -5.33 & -1.90 & -0.14 & 0.21 & 0.10 & $-0.73^{*}$ & -0.25 & -0.42 & 0.23 \\
\hline CSH3075 X H 1098-i & -4.42 & -1.83 & -0.42 & 0.23 & 0.06 & -0.19 & -0.38 & -0.15 & -0.08 \\
\hline CSH3075X H1117 & $14.17 *$ & $5.57 * *$ & 1.23 & $-0.33 * *$ & 0.01 & $0.67 *$ & 0.77 & $0.74 * *$ & -0.04 \\
\hline CSH3075X H1236 & -4.41 & -1.83 & -0.66 & -0.11 & -0.16 & 0.25 & -0.13 & -0.16 & -0.10 \\
\hline SE (d) & 5.75 & 1.98 & 0.63 & 0.12 & 0.11 & 0.34 & 0.50 & 0.23 & 0.12 \\
\hline
\end{tabular}

$* *$ Significant at $1 \%$ level of significance

*Significant at $5 \%$ level of significance 
Table.6 Above average specific cross combination for Different characters along with per se performance

\begin{tabular}{|c|c|c|c|c|}
\hline \multirow{2}{*}{ Characters } & \multicolumn{4}{|c|}{ Above average cross combination } \\
\hline & $\mathbf{1}^{\mathrm{st}}$ & Per se & $2^{\text {nd }}$ & Perse \\
\hline Seed cotton yield per plant (g) & $\begin{array}{c}\mathrm{AC} 726 \times \mathrm{H} 1236 \\
\left(27.76^{* *}\right)\end{array}$ & $\begin{array}{c}103.91 \\
(\mathrm{~g})\end{array}$ & $\begin{array}{c}\mathrm{H} 1476 \times \mathrm{H} 1226 \\
\left(26.19^{* *}\right)\end{array}$ & $\begin{array}{c}104.12 \\
(\mathrm{~g})\end{array}$ \\
\hline Lint yield per plant (g) & $\begin{array}{c}\mathrm{AC} 726 \times \mathrm{H} 1236 \\
(9.97 * *)\end{array}$ & $\begin{array}{c}34.91 \\
(\mathrm{~g})\end{array}$ & $\begin{array}{c}\mathrm{H} 1476 \times \mathrm{H} 1226 \\
\left(8.17^{* *}\right)\end{array}$ & $\begin{array}{c}33.70 \\
(\mathrm{~g})\end{array}$ \\
\hline Ginning outturn (\%) & $\begin{array}{c}\mathrm{H} 1463 \times \mathrm{H} 1236 \\
(1.78 * *)\end{array}$ & $\begin{array}{c}36.37 \\
(\%)\end{array}$ & $\begin{array}{c}\text { ISR12 x H1098-i } \\
\left(1.33^{*}\right)\end{array}$ & $\begin{array}{c}35.61 \\
(\%)\end{array}$ \\
\hline Seed index $(g)$ & $\begin{array}{c}\text { ISR12 x H1226 } \\
(0.94 * *)\end{array}$ & $\begin{array}{l}7.26 \\
(\mathrm{~g})\end{array}$ & $\begin{array}{c}\mathrm{H} 1476 \times \mathrm{H} 1098-\mathrm{i} \\
\left(0.56^{* *}\right)\end{array}$ & $\begin{array}{l}7.20 \\
(\mathrm{~g})\end{array}$ \\
\hline Lint index (g) & $\begin{array}{c}\text { ISR12 x H1226 } \\
\left(0.54^{* *}\right)\end{array}$ & $\begin{array}{c}3.84 \\
(\mathrm{~g}) \\
\end{array}$ & $\begin{array}{c}\mathrm{H} 1463 \times \mathrm{H} 1236 \\
(0.52 * *)\end{array}$ & $\begin{array}{c}4.08 \\
(\mathrm{~g})\end{array}$ \\
\hline $2.5 \%$ span length $(\mathrm{mm})$ & $\begin{array}{c}\text { HR1 } 1 \text {. H1117 } \\
\left(2.56^{* *}\right)\end{array}$ & $\begin{array}{l}26.23 \\
(\mathrm{~mm})\end{array}$ & $\begin{array}{c}\text { ISR12 x H1226 } \\
\left(2.26^{* *}\right)\end{array}$ & $26.53(\mathrm{~mm})$ \\
\hline Fibre uniformity (\%) & $\begin{array}{c}\text { HR1 x H1117 } \\
(0.85)\end{array}$ & $\begin{array}{c}80.33 \\
(\%)\end{array}$ & $\begin{array}{c}\text { CSH3075 x H1117 } \\
(0.77)\end{array}$ & $\begin{array}{c}81.00 \\
(\%)\end{array}$ \\
\hline Fibre strength (g/tex) & $\begin{array}{c}\text { ISR12 x H1226 } \\
(2.81 * *)\end{array}$ & $\begin{array}{l}28.20 \\
(\mathrm{~g} / \mathrm{tex})\end{array}$ & $\begin{array}{c}\mathrm{H} 1470 \times \mathrm{H} 1236 \\
\left(2.14^{* *}\right)\end{array}$ & $28.07(\mathrm{~g} / \mathrm{tex})$ \\
\hline Micronaire value ( $\mu \mathrm{g} /$ inch) & $\begin{array}{c}\mathrm{H} 1470 \times \mathrm{H} 1236 \\
\left(0.86^{* *)}\right)\end{array}$ & $4.50(\mu \mathrm{g} / \mathrm{inch})$ & H1476 x H1098-i (0.72**) & $4.30(\mu \mathrm{g} / \mathrm{inch})$ \\
\hline
\end{tabular}

SCA value in parenthesis

**Significant at $1 \%$ level of significance

*Significant at $5 \%$ level of significance 
Table.7 Estimation of economic heterosis in Gossypium hirsutum L

\begin{tabular}{|c|c|c|c|c|c|c|c|c|c|}
\hline Cross & $\begin{array}{c}\text { Seed cotton } \\
\text { yield/plant } \\
(\mathrm{g})\end{array}$ & $\begin{array}{c}\text { Lint } \\
\text { yield/plant } \\
(\mathrm{g})\end{array}$ & $\begin{array}{c}\text { Ginning } \\
\text { outturn }(\%)\end{array}$ & $\begin{array}{l}\text { Seed index } \\
(\mathrm{g})\end{array}$ & $\begin{array}{c}\text { Lint index } \\
(\mathrm{g})\end{array}$ & $\begin{array}{c}2.5 \% \text { span } \\
\text { length } \\
(\mathrm{mm})\end{array}$ & $\begin{array}{c}\text { Fibre } \\
\text { uniformity } \\
(\%)\end{array}$ & $\begin{array}{c}\text { Fibre } \\
\text { strength } \\
(\mathrm{g} / \mathrm{tex}) \\
\end{array}$ & $\begin{array}{c}\text { Micronaire } \\
\text { value ( } \mu \mathrm{g} / \text { inch })\end{array}$ \\
\hline H1156 X H1226 & -4.54 & $-15.58 * *$ & $-13.65^{*}$ & 2.36 & $-11.52 * *$ & -8.23 & -1.10 & 0.01 & $5.49 *$ \\
\hline H1156 X H 1098-i & 2.45 & $-18.00 * *$ & $-19.31 * *$ & -1.19 & -3.40 & -5.17 & -3.43 & -0.42 & -2.30 \\
\hline H1156 X H1117 & -0.35 & $-33.47 * *$ & $-34.78 * *$ & -1.99 & -4.06 & -6.92 & 1.37 & 0.42 & -1.53 \\
\hline H1156 X H1236 & 3.14 & $-19.40 * *$ & $-21.75 * *$ & -3.09 & $-7.55^{*}$ & $-11.82 *$ & -2.19 & 0.00 & -1.79 \\
\hline ISR12 X H1226 & 3.84 & $-39.21 * *$ & $-39.96 * *$ & -1.24 & 2.83 & 0.88 & $9.04 *$ & 0.83 & $7.91 *$ \\
\hline ISR12 X H 1098-i & 4.77 & $-21.24 * *$ & $-19.93 * *$ & 1.70 & $-10.48 * *$ & -8.14 & -2.47 & 0.42 & -2.81 \\
\hline ISR12 X H1117 & -2.91 & $-42.41 * *$ & $-44.10 * *$ & -2.89 & $-20.58 * *$ & $-23.99 * *$ & -2.47 & 0.00 & $-6.38 *$ \\
\hline ISR12 X H1236 & 2.79 & $-10.63 *$ & $-15.25 * *$ & -4.88 & $-11.99 * *$ & $-18.39 * *$ & 1.51 & 0.00 & -4.59 \\
\hline HR1 X H1226 & 2.21 & $-14.96^{*}$ & $-19.52 * *$ & $-5.39 *$ & $-14.39 * *$ & $-21.19 * *$ & $-8.22 *$ & -1.67 & $-9.18 *$ \\
\hline HR1 X H 1098-i & 0.12 & $-22.68 * *$ & $-24.36 * *$ & -2.22 & $-22.79 * *$ & $-25.39 * *$ & -1.78 & 0.42 & -0.26 \\
\hline HR1 X H1117 & 0.58 & $-13.62 *$ & $-17.00 * *$ & -3.92 & $-9.39 *$ & $-14.80 *$ & $7.81 *$ & 0.42 & 3.83 \\
\hline HR1 X H1236 & 0.58 & $-17.20 * *$ & $-19.71 * *$ & -3.07 & $-19.54 * *$ & $-23.21 * *$ & $-12.19 * *$ & -2.08 & $-13.52 * *$ \\
\hline Luxmi PKV X H1226 & 1.28 & -4.49 & -5.44 & -1.12 & $-19.59 * *$ & $-21.02 * *$ & 3.70 & 0.83 & $-6.51 *$ \\
\hline Luxmi PKV X H 1098-i & 0.93 & $-56.76 * *$ & $-58.27 * *$ & -3.47 & $-9.77 *$ & $-14.54 *$ & $7.95 *$ & 0.83 & -1.02 \\
\hline Luxmi PKV X H1117 & 1.28 & $-47.46 * *$ & $-46.84 * *$ & 1.06 & $-7.22 *$ & -5.69 & 2.47 & 0.42 & -4.08 \\
\hline Luxmi PKV X H1236 & $-26.09 * *$ & $-25.35 * *$ & 1.00 & $-13.31 * *$ & $-11.82 *$ & 0.96 & 0.42 & -4.21 & $-20.16 * *$ \\
\hline AC726 X H1226 & $-26.66 * *$ & $-27.58 * *$ & -1.24 & $-19.44 * *$ & $-21.02 * *$ & $-11.10 * *$ & -0.83 & $-11.86^{* *}$ & $-22.48 * *$ \\
\hline AC726 X H 1098-i & $-32.39 * *$ & $-37.36 * *$ & $-7.35^{*}$ & $-18.78 * *$ & $-27.58 * *$ & $-9.59 *$ & -1.25 & $-10.97 * *$ & $-12.40^{*}$ \\
\hline AC726 X H1117 & $-24.99 * *$ & $-25.32 * *$ & -0.46 & $-7.22 *$ & -7.79 & -3.29 & -0.42 & $-8.04 *$ & $-15.50 * *$ \\
\hline AC726 X H1236 & $22.57 * *$ & $25.12 * *$ & 2.17 & $-8.92 *$ & -5.87 & $-7.95^{*}$ & -1.25 & $-10.97 * *$ & $-20.16 * *$ \\
\hline Delta Pine X H1226 & $-39.25 * *$ & $-39.93 * *$ & -1.15 & $-16.33 * *$ & $-17.78 * *$ & -3.29 & 0.00 & $-7.27 *$ & $-17.05 * *$ \\
\hline Delta Pine X H 1098-i & $-40.52 * *$ & $-41.25 * *$ & -1.52 & $-19.68 * *$ & $-21.54 * *$ & -4.80 & 0.42 & -3.44 & 0.78 \\
\hline Delta Pine X H1117 & $-33.19 * *$ & $-36.07 * *$ & -4.30 & $-15.90 * *$ & $-21.37 * *$ & 1.51 & -0.42 & $-6.25^{*}$ & $-17.05 * *$ \\
\hline Delta Pine X H1236 & $-52.62 * *$ & $-51.47 * *$ & 2.45 & $-8.50 *$ & -4.99 & 4.38 & 0.83 & 2.93 & -4.65 \\
\hline H1472 X H1226 & $-26.56 * *$ & $-25.43 * *$ & 1.47 & -2.83 & -0.61 & -0.69 & -0.42 & 3.06 & -1.55 \\
\hline H1472 X H 1098-i & $-15.02 * *$ & $-12.85^{*}$ & 2.53 & $-8.54 *$ & -4.99 & 3.70 & 1.67 & $-7.53 *$ & $-15.50 * *$ \\
\hline H1472 X H1117 & -9.36 & $-14.79 *$ & $-5.94 *$ & $-6.94 *$ & $-15.24 * *$ & 3.43 & 0.83 & 2.42 & -4.65 \\
\hline H1472 X H1236 & $-16.58 * *$ & $-14.70^{*}$ & 2.17 & -0.33 & 3.24 & 2.88 & 0.83 & 1.79 & -5.43 \\
\hline H1465 X H1226 & $-14.14 *$ & $-12.21 *$ & 2.28 & 0.80 & 4.47 & 2.33 & -0.42 & 1.91 & 4.65 \\
\hline
\end{tabular}




\begin{tabular}{|c|c|c|c|c|c|c|c|c|c|}
\hline H1465 X H 1098-i & 1.88 & -0.78 & -1.82 & 1.75 & -1.05 & $7.12^{*}$ & 0.83 & -3.44 & $-12.40^{*}$ \\
\hline H1465 X H1117 & $-22.58 * *$ & $-20.96 * *$ & 2.08 & -3.26 & -0.18 & 3.70 & 0.83 & $-6.00 *$ & -8.53 \\
\hline H1465 X H1236 & -7.59 & -9.88 & -3.28 & -4.20 & -9.02 & 4.11 & 0.83 & $-5.87 *$ & $-17.83^{* *}$ \\
\hline H1463 X H1226 & 1.45 & -5.20 & $-6.63 *$ & $-5.57 *$ & $-14.97 *$ & -1.23 & 0.42 & $-7.65^{*}$ & $17.05^{* * *}$ \\
\hline H1463 X H 1098-i & -3.36 & -7.12 & -3.77 & $-18.88 * *$ & $-23.47 * *$ & $7.12 *$ & 0.83 & $7.27 *$ & -1.55 \\
\hline H1463 X H1117 & $-36.05 * *$ & $-36.76 * *$ & -1.29 & $-5.57 *$ & -7.44 & 1.51 & 0.42 & 2.68 & 8.53 \\
\hline $\mathrm{H} 1463 \times \mathrm{H} 1236$ & $-15.95 * *$ & $-12.76^{*}$ & 3.88 & 1.04 & 7.18 & -4.66 & 0.42 & -3.06 & -3.88 \\
\hline H1464 x H1226 & 0.86 & -0.67 & -1.38 & $-6.23 *$ & -8.14 & 3.29 & 0.83 & -2.68 & $-12.40 *$ \\
\hline H1464 X H 1098-i & $-27.73 * *$ & $-30.39 * *$ & -3.68 & 4.01 & -1.75 & $12.47 * *$ & 2.08 & $11.74 * *$ & -1.55 \\
\hline H1464 X H1117 & 0.21 & -4.38 & -4.52 & -1.42 & -8.14 & $8.36^{*}$ & 0.83 & $7.53 *$ & 0.78 \\
\hline H1464 X H1236 & $12.65^{*}$ & $15.37 * *$ & 2.38 & $5.47 *$ & 9.28 & 4.52 & 0.83 & 2.93 & -5.43 \\
\hline H1470 X H1226 & 0.09 & -1.31 & -1.48 & $-8.73^{*}$ & $-10.77^{*}$ & -0.55 & 0.42 & $-8.16^{*}$ & $-26.36 * *$ \\
\hline H1470 X H 1098-i & $37.84 * *$ & $36.62 * *$ & -0.76 & $-8.26^{*}$ & -9.28 & $6.16^{*}$ & 0.83 & -2.30 & $-21.71 * *$ \\
\hline H1470 X H1117 & 8.24 & 9.97 & 1.54 & 3.40 & 5.78 & $8.90^{*}$ & 1.25 & -0.26 & $-14.73^{*}$ \\
\hline H1470 X H1236 & $45.04 * *$ & $38.28 * *$ & -4.67 & 3.02 & -4.20 & $5.34^{*}$ & 1.25 & $7.40^{*}$ & 4.65 \\
\hline H1471 X H1226 & $-24.32 * *$ & $-24.17 * *$ & 0.30 & -0.99 & -0.53 & 2.06 & -0.42 & -3.06 & $-24.03 * *$ \\
\hline H1471 X H 1098-i & -7.65 & -4.68 & 3.29 & -0.61 & 4.47 & $7.53^{*}$ & 0.83 & -1.66 & $-17.83^{* *}$ \\
\hline H1471X H1117 & -4.97 & -2.23 & 2.95 & $-7.50^{*}$ & -3.24 & -2.19 & -0.42 & -1.91 & 3.88 \\
\hline H1471 X H1236 & $-21.77 * *$ & $-18.17 * *$ & 4.55 & 3.30 & $10.68^{*}$ & $6.03^{*}$ & 0.83 & $5.36^{*}$ & 4.65 \\
\hline H1476 X H1226 & $22.82 * *$ & $20.78^{*}$ & -1.74 & $-6.61^{*}$ & -9.02 & $5.48^{*}$ & 0.83 & -0.77 & $-20.93^{* * *}$ \\
\hline H1476 X H 1098-i & $-11.60^{*}$ & $-10.21 *$ & 1.03 & 1.98 & 3.50 & $5.89 *$ & 0.83 & $7.14 *$ & 0.00 \\
\hline H1476 X H1117 & $-21.34 * *$ & $-20.70 * *$ & 0.74 & $-10.85 * *$ & -9.90 & -2.06 & 0.42 & $-5.23 *$ & $-27.13^{* * *}$ \\
\hline H1476 X H1236 & $-25.70 * *$ & $-26.21 * *$ & -0.92 & -3.45 & -4.73 & 2.74 & 0.42 & -4.85 & $-20.16^{* * *}$ \\
\hline H1477 X H1226 & $-40.92 * *$ & $-40.53 * *$ & -0.77 & -2.88 & -4.03 & -0.96 & 0.00 & $-6.38^{*}$ & $-12.40 *$ \\
\hline H1477X H 1098-i & $-48.20 * *$ & $-47.01 * *$ & 2.33 & $-9.30 *$ & -6.04 & -0.96 & 0.42 & $-6.25^{*}$ & $-16.28^{*}$ \\
\hline H1477 X H1117 & $-28.75^{* *}$ & $-29.63^{* *}$ & -1.24 & -2.88 & -4.73 & -0.27 & 0.00 & 2.04 & 2.33 \\
\hline H1477 X H1236 & $-28.44 * *$ & $-27.50 * *$ & 1.21 & $-6.65^{*}$ & -4.99 & $9.45^{*}$ & 0.83 & 2.42 & $-12.40^{*}$ \\
\hline CSH3075 X H1226 & $-26.77 * *$ & $-25.34 * *$ & 1.94 & $-6.98 *$ & -4.12 & -4.25 & -0.42 & $-9.31 *$ & $-12.40^{*}$ \\
\hline CSH3075 X H 1098-i & $-28.79 * *$ & $-28.08 * *$ & 1.16 & $-7.50 *$ & -5.78 & 0.69 & 0.00 & $-6.12 *$ & $-20.16^{* *}$ \\
\hline CSH3075X H1117 & -8.45 & -2.95 & $6.00^{*}$ & $-14.39 * *$ & -6.31 & 4.11 & 1.25 & -2.81 & $-19.38^{* *}$ \\
\hline CSH3075X H1236 & $-21.37 * *$ & $-20.01 * *$ & 1.46 & $-9.11 *$ & -7.01 & 1.23 & 0.00 & $-6.89 *$ & $-21.71 * *$ \\
\hline Minimum & -56.76 & -58.27 & -7.35 & -22.79 & -27.58 & -12.19 & -2.08 & -13.52 & -29.46 \\
\hline Maximum & 45.04 & 38.28 & 6.00 & 5.47 & 10.68 & 12.47 & 2.08 & 11.74 & 17.05 \\
\hline
\end{tabular}


Table.8 Estimates of economic heterosis (\%) of five better performing crosses for different traits

\begin{tabular}{|c|c|c|c|c|c|c|c|c|c|}
\hline $\begin{array}{l}\text { Best } \\
\text { cross }\end{array}$ & $\begin{array}{c}\text { Seed } \\
\text { cotton } \\
\text { yield/plant } \\
(\mathrm{g})\end{array}$ & $\begin{array}{c}\text { Lint } \\
\text { yield/plant } \\
\text { (g) }\end{array}$ & $\begin{array}{c}\text { Ginning } \\
\text { outturn } \\
(\%)\end{array}$ & $\begin{array}{c}\text { Seed } \\
\text { index }(g)\end{array}$ & $\begin{array}{c}\text { Lint } \\
\text { index }(g)\end{array}$ & $\begin{array}{c}2.5 \% \\
\text { span } \\
\text { length } \\
(\mathrm{mm})\end{array}$ & $\begin{array}{c}\text { Fibre } \\
\text { uniformity } \\
(\%)\end{array}$ & $\begin{array}{c}\text { Fibre } \\
\text { strength } \\
(\mathrm{g} / \mathrm{tex})\end{array}$ & $\begin{array}{c}\text { Micronaire } \\
\text { value } \\
(\mu \mathrm{g} / \text { inch })\end{array}$ \\
\hline 1. & $\begin{array}{c}\mathrm{H} 1470 \mathrm{x} \\
\mathrm{H} 1236 \\
(45.04)\end{array}$ & $\begin{array}{c}\text { H1470 x } \\
\text { H1236 } \\
(38.28)\end{array}$ & $\begin{array}{c}\text { CSH3075 } \\
\text { x H1117 } \\
(6.00)\end{array}$ & $\begin{array}{c}\text { H1464 x } \\
\text { H1236 } \\
(5.47) \\
\end{array}$ & $\begin{array}{c}\text { H1471 x } \\
\text { H1117 } \\
(10.68) \\
\end{array}$ & $\begin{array}{c}\text { H1464 x } \\
\text { H1098-I } \\
(12.47) \\
\end{array}$ & $\begin{array}{c}\text { H1464X } \\
\text { H } 1098-\mathrm{i} \\
(2.08) \\
\end{array}$ & $\begin{array}{c}\text { H1464 x } \\
\text { H1098-i } \\
(11.76)\end{array}$ & $\begin{array}{c}\mathrm{H} 1463 \mathrm{x} \\
\mathrm{H} 1226 \\
(17.05)\end{array}$ \\
\hline 2. & $\begin{array}{c}\text { H1470 x } \\
\text { H1098-i } \\
(37.84)\end{array}$ & $\begin{array}{c}\mathrm{H} 1470 \mathrm{x} \\
\mathrm{H} 1098-\mathrm{i} \\
(36.62)\end{array}$ & $\begin{array}{c}\text { H1471 x } \\
\text { H1236 } \\
(4.55) \\
\end{array}$ & $\begin{array}{c}\mathrm{H} 1471 \mathrm{x} \\
\mathrm{H} 1236 \\
(4.55) \\
\end{array}$ & $\begin{array}{c}\mathrm{H} 1464 \mathrm{x} \\
\mathrm{H} 1236 \\
(9.28) \\
\end{array}$ & $\begin{array}{c}\text { H1477 x } \\
\text { H1236 } \\
(9.45) \\
\end{array}$ & $\begin{array}{c}\mathrm{H} 1472 \times \\
\mathrm{H} 1098-\mathrm{i} \\
(1.67)\end{array}$ & $\begin{array}{c}\text { ISR12 x } \\
\text { H1236 } \\
(7.91) \\
\end{array}$ & $\begin{array}{c}\mathrm{H} 1463 \times \\
\mathrm{H} 1117 \\
(8.53)\end{array}$ \\
\hline 3. & $\begin{array}{c}\text { H1476 x } \\
\text { H1226 } \\
(22.82)\end{array}$ & $\begin{array}{c}\text { AC726 x } \\
\text { H1236 } \\
(25.12)\end{array}$ & $\begin{array}{c}\text { H1463 x } \\
\text { H1236 } \\
(3.87)\end{array}$ & $\begin{array}{c}\text { H1464 x } \\
\text { H1098-I } \\
(3.40)\end{array}$ & $\begin{array}{c}\mathrm{H} 1463 \mathrm{x} \\
\mathrm{H} 1236 \\
(7.18)\end{array}$ & $\begin{array}{c}\text { ISR12 x } \\
\text { H1226 } \\
(9.04)\end{array}$ & $\begin{array}{c}\mathrm{H} 1470 \times \\
\mathrm{H} 1117 \\
(1.25)\end{array}$ & $\begin{array}{c}\mathrm{H} 1464 \times \\
\mathrm{H} 1117 \\
(7.53)\end{array}$ & $\begin{array}{c}\text { H1156 X } \\
\text { H1226 } \\
(6.20)\end{array}$ \\
\hline 4. & $\begin{array}{c}\text { AC726 x } \\
\text { H1236 } \\
(22.57)\end{array}$ & $\begin{array}{l}\text { H1476 x } \\
\text { H1226 } \\
(20.78)\end{array}$ & $\begin{array}{c}\mathrm{H} 1471 \times \mathrm{H} \\
1098-\mathrm{I} \\
(3.29)\end{array}$ & $\begin{array}{c}\mathrm{H} 1471 \mathrm{x} \\
\mathrm{H} 1236 \\
(3.30)\end{array}$ & $\begin{array}{c}\text { H1464 x } \\
\text { H1098-I } \\
(5.78)\end{array}$ & $\begin{array}{c}\mathrm{H} 1470 \mathrm{x} \\
\mathrm{H} 1117 \\
(8.91)\end{array}$ & $\begin{array}{c}\mathrm{H} 1470 \times \\
\mathrm{H} 1236 \\
(1.25)\end{array}$ & $\begin{array}{c}\mathrm{H} 1470 \times \\
\mathrm{H} 1236 \\
(7.40)\end{array}$ & $\begin{array}{c}\mathrm{H} 1465 \mathrm{x} \\
\mathrm{H} 1226 \\
(4.65)\end{array}$ \\
\hline 5. & $\begin{array}{c}\text { H1464 x } \\
\text { H1236 } \\
(12.64)\end{array}$ & $\begin{array}{c}\text { H1464 x } \\
\text { H1236 } \\
(15.36)\end{array}$ & $\begin{array}{c}\text { H1471 x } \\
\text { H1117 } \\
(2.95)\end{array}$ & $\begin{array}{c}\mathrm{H} 1470 \mathrm{x} \\
\mathrm{H} 1236 \\
(3.02)\end{array}$ & $\begin{array}{c}\text { H1471 x } \\
\text { H } 1098-\mathrm{I} \\
\quad(4.67)\end{array}$ & $\begin{array}{c}\text { H1464 x } \\
\text { H1117 } \\
(8.36)\end{array}$ & $\begin{array}{c}\text { CSH3075 } \\
\times \text { H1117 } \\
(1.25)\end{array}$ & $\begin{array}{c}\mathrm{H} 1463 \times \\
\mathrm{H} 1098-\mathrm{i} \\
(7.27)\end{array}$ & $\begin{array}{c}\mathrm{H} 1470 \mathrm{x} \\
\mathrm{H} 1236 \\
(4.65)\end{array}$ \\
\hline
\end{tabular}

The hybrid HR1 x H1117 found highest specific combining ability for $2.5 \%$ span length and uniformity ratio while, hybrid ISR12 x H1226 was good specific combiner for fibre strength indicated both the crosses were combinations of poor $\mathrm{x}$ good and good $\mathrm{x}$ poor combining parents; hence, SCA effect of these crosses is due to both additive and nonadditive gene action. The hybrid H1470 x H1236 reported highest specific combination for micronaire value, which was a combination of poor $\mathrm{x}$ poor general combiner parents indicating that non-additive type gene action to be more important for improvement of this character. These findings are in confirmation with the findings of Karademr and Gencer (2010), Anandan (2010) and Sawarkar et al., (2015).

The values for the heterosis of individual crosses were recorded for all nine characters presented in table 7. Five superior hybrids selected based on economic heterosis have been listed in table 8. Several crosses were found to possess considerable amount of economic heterosis for the seed cotton yield and fibre quality traits. Eleven crosses observed positive heterotic value for seed cotton yield and out of which 4 hybrids exhibited heterotic values of more than 20 per cent. Among these hybrids, five hybrids H1470 x H1236 (45.04\%) followed by H1470 x H1098-i (37.84\%), H1476 x H1226 (22.82\%), AC726 x H1236 (22.57\%) and H1464 x H1236 (12.64\%) exhibited positive and best heterotic effects over the commercial check HHH 223 for seed cotton yield. Heterosis for seed cotton yield and other related characters in upland cotton has also been reported earlier by Rajamani et al., (2009), Patil et al., (2011), Jaiwar et al., (2012), Sawarkar et al., (2015) and Sharma et al., (2016).

Maximum heterosis over check HHH223 for lint yield was recorded in crosses $\mathrm{H} 1470 \mathrm{x}$ H1236 (38.28\%), H1470 x H 1098-i (36.62\%) and AC726 x H1236 (25.12\%). All the better performing hybrids for lint yield revealed high heterosis for seed cotton yield exhibiting high correlation between these two characters. For ginning out turn the highest heterotic value was recorded for the cross CSH3075 x H1117 (6.00\%), H1471 x H1236 (4.55\%) and 
H1463 x H1236 (3.87\%). Similar result was found by Khan et al., (2010), Patil et al., (2011), Tuteja (2014) and Deosarkar et al., (2014).

For seed index, the higher economic heterosis was observed in H1464 x H1236 (5.47\%), followed by $\mathrm{H} 1464 \times \mathrm{H} 1098-\mathrm{i}(3.40 \%)$ and H1471 x H1236 (3.30\%). Out of sixty crosses, ten crosses exhibited positive economic heterotic effects for seed index, while for lint index maximum heterotic value over standard check were recorded in crosses H1471 x H1117 (10.68\%) followed by H1464 x H1236 (9.28\%) and H1463 x H1236 $(7.18 \%)$. However, in general the heterotic value for lint index obtained a marked increase in seed index. Similar results were reported by Hussain et al., (2009), Basal et al., (2011) and Sawarkar et al., (2015).

The crosses, namely H1464 x H1098-I (12.47), H1477 x H1236 (9.45) and ISR12 x H1226 (9.04) revealed higher heterosis over standard check for $2.5 \%$ span length. The hybrids H1464 X H 1098-i (2.08) and H1472 $\times$ H1098-i (1.67) observed maximum economic heterotic effects for fibre uniformity. The highest economic heterosis for fibre strength was reported from the crosses H1464 x H1098-i (11.76), ISR12 x $\mathrm{H} 1236$ (7.91) and H1464 × H1117 (7.53). Basis of the three important fibre quality traits like $2.5 \%$ span length, fibre uniformity and fibre strength the best heterotic combination reported from the cross H1464 x H1098-i. The maximum heterotic effect for micronaire value was observed by the hybrids H1463 x H1226 (17.05), H1463 × H1117 (8.53) and H1156 $\times$ H1226 (6.20). Varying magnitude of heterosis for fibre quality traits has been reported in G. hirsutum by several workers Patil et al., (2012) and Kaliyaperumal et al., (2013). The present study revealed that the ratio of $\delta^{2}$ GCA $/ \delta^{2}$ SCA was less than unity for all the nine characters indicating preponderance of non-additive gene action (dominance and epistasis), which is an important in exploitation of heterosis through hybrid breeding. The best general combining ability was detected from the parent H1470 for seed cotton yield, H1464 and H1098-i for fiber quality traits. SCA was significant for AC726 x H1236, ISR12 x H1226, HR1 x H1117 hybrid combinations for yield and fiber quality. The crosses H1470 x H1236 and H1470 x H1098-i were reported good heterosis for seed cotton yield as well as for fibre quality selected the best hybrids were H1464 x H1098-i and H1463 x H1226. The cross combination involving H1470, H1098-i and H1464 parents' recorded significant positive heterosis with acceptable SCA effect for both yield and fibre quality parameters. This study suggested that the parents H1470, H1098-i and H1464 can be used in hybrid development programme with better fibre quality. The result has been discussed in the light of their usefulness in future breeding programs.

\section{References}

Ahuja, S.L. and Dhayal, L.S. 2007. Combining ability estimates for yield and fibre quality traits in $4 \times 13$ line $\mathrm{x}$ tester crosses of Gossypium hirsutum. Euphytica, 153(2): 87-98.

Alkuddsi, Y.A., Rao, M.R., Patil, S.S., Gowda, T.H. and Joshi, M. 2013. Combining ability analysis for seed cotton yield and its components in intra hirsutum hybrids and forming heterotic boxes for exploitation in cotton. Genomics and Applied Biology, 4(5): 35-49.

Anandan, A. 2010. Environmental impact on the combining ability of fiber traits and seed cotton yield in cotton. Journal of Crop Improvement, 24(4): 310-323.

Basal, H., Canavar, O., Khan, N.U. and Cerit, C.S. 2011. Combining ability and 
heterotic studies through line $\mathrm{x}$ tester in local and exotic upland cotton genotypes. Pakistan Journal of Botany, 43(3): 1699-1706.

Deosarkar, D.B., Deshmukh, J.D. and Deshmukh, V.D. 2014. Combining ability analysis for yield and fibre quality traits in upland cotton (Gossypium hirsutum L.). Journal of Cotton Research and Development, 28(1): $18-23$.

Deshmukh, J.D., Deosarkar, D.B. and Deshmukh, V.D. 2014. Heterosis for fibre quality traits in cotton (Gossypium hirsutum L). Journal of Cotton Research and Development, 28(2): 217219.

Hussain, K., Abbas, G., Aslam, M., Hussnain, H., Akhtar, M.N. and Irshad, M. 2009. Heterosis and inbreeding depression estimates for yield and fibre components in upland cotton ( $G$. hirsutum L.). International Journal of Biology and Biotechnology, 6(4): 233236.

Jaiwar, S.S., Avinashe, H.A. and Patel, B.N. 2012. Heterosis for seed cotton yield and its contributing traits in upland cotton (G. hirsutum L.). Journal Soils and Crops, 22(2): 314-320.

Kaliyaperumal, A.K., Karuppanasamy, S.K. and Ravikesavan, R. 2013. Heterosis studies for fibre quality of upland cotton in line $\mathrm{x}$ tester design. African Journal of Agricultural Research, 8(48): 63596365.

Karademir, C., Gencer, O. and Karademir, E. 2007. Heterosis and combining ability for yield and fiber properties in cotton (Gossypium hirsutum L.) under drought stress conditions. Asian Journal of Plant Science, 6(4): 667-672.

Karademr, E. and Gencer, O. 2010. Combining ability and heterosis for yield and fiber quality properties in cotton ( $G$. hirsutum L.) obtained by half diallel mating design. Notulae Botanicae Horti Agrobotanici ClujNapoca, 38(1): 222-227.

Kempthorne, O. 1957. An Introduction to Genetic Statistics, New York, John Wiley and Sons, $1^{\text {st }}$ Edition, pp: 456471.

Kencharaddi, H.G., Hanchinal, R.R. and Patil, S.S. 2015. Studies on combining ability in inter heterotic group derived cotton hybrids for lint yield and its components. Research on Environment and Life Science, 8(3): 451-456.

Khan, M.A., Iqbal, M. and Jamil, M. 2010. Finding heterosis for fibre traits in intervarietal crosses of cotton ( $G$. hirsutum L.). Frontiers of Agriculture in China, 4(2): 185-187.

Khan, N.U., Marwat, K.B., Gul H., Kumbhar, M.B., Farhatullah, Soomro, Z.A., Khan, N., Aisha Parveen and Umm-e-Aiman. 2009. Study of fiber quality traits in upland cotton using additive-dominance model. Pakistan Journal of Botany, 41(3): 1271-1283.

Kumar, M., Nirania, K.S., Sangwan, R.S. and Yadav, N.K. 2013. Combining ability studies for yield and quality traits in upland cotton (Gossypium hirsutum L.). Journal of Cotton Research and Development, 27(2): 171-174.

Nidagundi, J.M., Deshpande, S.K., Patil, B.R. and Mane, R.S. 2011. Combining ability and heterosis for yield and fibre quality traits in American cotton. Crop Improvement, 38(2): 179-185.

Panse, V.G. and Sukhatme, P.V. 1961. Statistical methods for agricultural workers. Indian Council of Agricultural Research, New Delhi. Pp. 381.

Patil, S.A., Naik, M.R., Pathak, V.D. and Kumar, V. 2012. Heterosis for yield and fibre properties in upland cotton (Gossypium hirsutum L.). Journal of Cotton Research and Development, 26(1): 26-29. 
Patil, S.A., Naik, M.R., Chaugule, G.R., Pathak, V.D. and Patil, A.B. 2011. Combining ability analysis for yield and fibre quality traits in upland cotton (Gossypium hirsutum L.). Journal of Cotton Research and Development, 25(2): 171-175.

Pushpam, R., Thangaraj K. and Raveendran T.S. 2015. Heterosis and combining ability studied in upland cotton for yield characters. Electronic Journal of Plant Breeding, 6(2): 459-463.

Rajamani, S., Gopinath, M. and Reddy, K.H.P. 2014. Combining ability for seed cotton yield and fibre characters in upland cotton (Gossypium hirsutum L.). Journal of Cotton Research and Development, 28(2): 207-210.

Rajamani, S., Rao, C.M. and Naik, R.K. 2009. Heterosis for yield and fibre properties in upland cotton (Gossypium hirsutumL.). Journal of Cotton Research and Development, 23(1): 4345.
Sawarkar, M., Solanke, A., Mhasal, G.S. and Deshmukh, S.B. 2015. Combining ability and heterosis for seed cotton yield, its components and quality traits in Gossypium hirsutum L. Indian Journal of Agriculture Science, 49(2): 154-159.

Sharma, R., Gill, B.S. and Pathak, D. 2016. Heterobeltiosis for yield, its component traits and fibre properties in upland cotton (Gossypium hirsutum L.). Journal of Cotton Research and Development, 30(1): 11-15.

Subramanian, A., Ravikesvan, R., Lyanar, K. and Vindhavarman, P. 2005. Combining ability analysis in upland cotton (Gossypium hirsutum L.). Plant Archives, 5(1): 23-28.

Tuteja, O.P. 2014. Studies on heterosis for yield and fibre quality traits in GMS hybrids of upland cotton (Gossypium hirsutum L.). Journal of Cotton Research and Development, 28(1): 1-6.

\section{How to cite this article:}

Sukhdeep Singh Sivia, S.S. Siwach, O. Sangwan and Sunayana. 2017. Development of Superior Hybrids for Fibre Quality based on Heterosis and Combining Ability in Upland Cotton (Gossypium hirsutum L.). Int.J.Curr.Microbiol.App.Sci. 6(8): 448-461. doi: https://doi.org/10.20546/ijcmas.2017.608.059 